\title{
Tres momentos de institucionalización de la educación de anormales en Colombia: análisis desde las políticas educativas
}

\author{
Gloria Janneth Orjuela Sánchez ${ }^{1}$
}

Recibido: 10-02-2017

Aceptado: 07-03-2017

\section{RESUMEN}

Se pretende mostrar aquí una primera forma de acercamiento a la noción de diferencia desde las políticas educativas, con lo que se pretende evidenciar los momentos que han tenido estas políticas, en relación con las formas de institucionalización de la educación para la diferencia en Colombia. En esa línea de ideas, cabe anotar que se hará uso de la historia como instrumento que permitirá analizar la política, sus reformas, sus efectos y la relación de ésta con la noción de diferencia. Así mismo, es la historia la que posibilitará poner en diálogo reflexiones acerca de la inclusión y la educación de la diferencia.

Palabras clave: diferencia, política educativa, institucionalización, inclusión. 


\title{
Three moments of institutionalization of the education of abnormal ones in Colombia: Analysis from educational policies
}

\begin{abstract}
Is intended to show a first form of approach to the notion of difference here from the educational policies,-when we talk about the difference do not in the sense of opposition to the same, then the difference would be on the side of what is repeated, in where this does not necessarily always be equal-. That is, demonstrate the moment that has had this policy, in relation the forms of institutionalization of education for the difference in Colombia. In that line of ideas, it should be noted that there will be use of history as a tool allowing analyzing policy, its reforms, its effects and its relationship with the notion of difference. Likewise, it is history which will make it possible put into dialogue reflections about the inclusion and education of the difference.
\end{abstract}

Keywords: Difference, education policy, institutionalization, inclusion.

\section{Introducción}

El interés por la anormalidad y el ejercicio educativo que se ha llevado a cabo con estas poblaciones, responde a dos momentos del ejercicio del biopoder ${ }^{2}$ : uno anatomopolítico, concentrado en el sujeto y la

2. Un poder que se preocupará por administrar la vida y sus formas de actuación. Sus prácticas están situadas en dos momentos concretos y con maneras de actuación particulares: uno, de actuaciones disciplinarias: anatomopolítica del cuerpo humano; y el otro en la forma de control y regulación: biopolítica de la población. (Para ampliación del concepto revisar Foucault, 2007, capítulo: "Derecho de muerte y poder sobre la vida". 
homogenización, poder de normalización ${ }^{3}$; estudio disciplinar de observación, medición y clasificación de los cuerpos que integrará para normalizar. El otro, biopolítico, donde ya no es el centro el sujetoindividuo, sino el sujeto-especie, el grupo; un poder inclusivo, potenciador de grupos vulnerables4: la inclusión, la multiculturalidad; se evidenciará en el respeto y potenciación de las diferencias. Incluye para gobernar, para administrar y potenciar.

El tránsito del uno al otro se da por un giro que descentra o cambia la mirada y el interés: del primero concentrado en el suojeto, el individuo, el cuerpo a normalizar u homogenizar; se pasará al segundo, interesado por la especie, el grupo, el ambiente, el medio y los apoyos que este provea. Pero es un giro que estará mediado por una serie de tensiones o condiciones que le dan posibilidad; es decirno se da de manera automática, causal o como si el uno fuese consecuencia del otro. Cada cual responde a unas condiciones particulares -esto les da una impronta histórica-, pero además estos momentos tendrán unos efectos particulares sobre las formas de concepción del otro -anormal, diferente-, sobre los saberes que se ponen a funcionar; sobre las formas de visibilidad que se adecuan y sobre las formas de ejercicio de la gubernamentalidad o de gobierno de los otros.

En últimas, podría pensarse en una racionalidad (biopoder) con dos momentos -o formas de explicación- hacia la diferencia; racionalidad e instantes en los que se vinculan o articulan unas formas de saber

3. El poder de normalización: aquel que, según muestra Foucault en Los Anormales, se constituirá en Europa, hacia mediados del siglo XVIII como resultado del cruce entre el discurso de la psiquiatría médica y la práctica judicial -no discursiva-, conformando lo que el autor denominará las pericias médico-judiciales. Estas buscaban básicamente homogenizar e institucionalizar para ejercer técnicas de expiación y terapéutica sobre sujetos perversos, peligrosos. La pericia médico-judicial será el mecanismo de unión entre el discurso médico y el judicial con efectos precisos en la necesidad de un experto, es decir uno de los efectos es la creación de una práctica que se dirigirá a la categoría de los anormales o al campo de gradación de lo normal a lo anormal; constituyéndose de esa manera en el poder de normalización. La noción central (y a la vez función central) que este poder manejará, por lo menos desde sus prácticas institucionalizadas -como en la escuela-, será la de homogenización, mecanismo que funcionará a partir del disciplinamiento, la docilidad, la observación, la regularización, la distribución y la inclusión de individualidades diferenciales. Será este un modelo de control inclusivo, potenciador y productor. Un poder siempre ligado a un saber: el arte de gobernar, el ejercicio de un gobierno que desde la edad clásica introdujo un aparato: el Estado, y al lado de él, diferentes instituciones; a los que se les transferirá parte de la técnica general del ejercicio del poder: del gobierno de los hombres, una de ellas será la escuela, que se constituirá en uno de los dispositivos de este ejercicio como una organización disciplinaria que tendrá como efecto directo, justamente la normalización.

4. Desde el Ministerio de Educación Nacional (MEN) se identifica a las poblaciones vulnerables como aquellas "... personas que, por su naturaleza o por determinadas circunstancias, se encuentran en mayor medida expuestos a la exclusión, la pobreza y los efectos de la inequidad y la violencia de todo orden". En ese sentido la vulnerabilidad será entendida en el país y en el sector educativo como: "un fenómeno que deteriora el bienestar y la calidad de vida de las personas y que retrasa el desarrollo de los pueblos" (2005, p. 5). 
con unas formas de poder permitiendo que se institucionalicen varios momentos de la educación de anormales o de la diferencia en Colombia. El paso de una etapa anatomopolítica a una biopolítica no será, de todas formas, homogéneo ni unificado en el plano nacional; en ese sentido, conviene insistir en que esos dos "momentos" se traslapan o conviven, en esa medida, también es probable encontrar alguna dispersión en sus manifestaciones.

Para el caso de este artículo se evidenciarán tres instantes en la política educativa colombiana, a través del trazado de un mapa de la diferencia que ubicará periodos y condiciones posibilitadoras. Se busca con ello problematizar y/o profundizar el sentido y las implicaciones en el cambio de las estrategias de gobierno de la diferencia para el mundo de hoy.

\section{Primer momento: la civilización determina un tipo de diferencia desde las carencias y desigualdades}

La primera mitad del siglo XX es coincidente con el discurso de la Pedagogía Activa que situaba al niño como el centro de la educación; con planteamientos de la medicina que empiezan a determinar lo patológico, una gradación de lo normal a lo anormal y con una forma de gubernamentalidad o ejercicio de gobierno que pretendía regenerar, purificar la raza -desde la educación- mediante estrategias puestas a funcionar en la escuela; forma disciplinar de gobierno, concentrado en un tipo de poder de normalización y estrategias anatomo-políticas que buscaban el disciplinamiento, la ortopédica o corrección. ¿Es el momento de ingreso de la educación para la diferencia en la escuela colombiana?

En efecto, la infancia va a aparecer con fuerza en el panorama nacional, considerada fundamental para el futuro de la modernidad, entrará en el escenario social y educativo de la mano de la pedagogía, de algunos saberes modernos como la psicología, la medicina, la fisiología, etc., que, en manos de intelectuales, políticos, maestros preocupados por el futuro de la raza, cobraran vida en la escuela.

Además, está presente y vigente la polémica alrededor de la degeneración racial que pondrá la sociedad en total revuelo, asumiendo diversidad de posturas. Unas, radicales: olvidarse, allí no hay nada que 
hacer; es necesario mezclar la raza con nuevos aires, por ejemplo, del continente europeo y anglosajón preferiblemente; otras, parciales: pensar que mediante la higiene, la enseñanza, el ingreso a la escuela, podría regenerarse y hacer posible el futuro de progreso y modernización. Radicales o parciales, dichas posturas tuvieron por efecto una suerte de acciones como: reglamentar el funcionamiento de las escuelas; establecer la enseñanza de la higiene ${ }^{5}$; la creación de un Ministerio de Instrucción y Salubridad Públicas; llevar el médico a la escuelas, entre otras.

Para el caso colombiano, la Instrucción pública se rigió por los principios del Decreto Orgánico de Instrucción Pública de 1870. Esta política educativa sólo hasta la década del 70 del siglo XX será sustancialmente modificada, a excepción de algunas reformas que procuraron introducir cambios en el sector educativo, coincidiendo con la Reforma Liberal de los 30. Es decir que es la Ley Orgánica de Instrucción Pública la política educativa que rigió los destinos de la educación del país en este período y junto con ella el Decreto que la reglamentó; de estos documentos, y con fines del rastreo que interesa para esta ponencia, vale la pena destacar:

La creación de un sistema de inspección nacional, departamental y local ejercido tanto sobre maestros y alumnos, como sobre funcionarios, que tendrá fuerte concentración en la vigilancia del sistema de alimentación, dormitorios ${ }^{6}$, faltas de disciplina cometidas dentro y fuera de la escuela; además le corresponderá estar al tanto del cumplimiento de las condiciones esenciales para el desarrollo físico y moral de los alumnos; igualmente será el sistema de inspección en cabeza del funcionario nombrado para tal fin, el encargado de hacer efectivas las multas o responsabilidades frente a descuidos $u$ omisiones ${ }^{7}$. Se informará así mismo sobre las regulaciones, la disciplina, la salubridad, la conducta de los alumnos, el sistema

5. Ley 19260012 (septiembre 25) "Sobre enseñanza de la higiene, saneamiento de los puertos marítimos, fluviales y terrestres y de las principales ciudades de la República". Publicada por el Diario oficial 20307 lunes 27 de septiembre de 1926.

6. "Art. 41. Todo Establecimiento oficial o particular, tenga o no internado, estará sometido a la inspección del Gobierno en lo tocante al sistema de alimentación, vigilancia de dormitorios y demás condiciones esenciales relativas al desarrollo físico y moral de los alumnos. El Ministerio de Instrucción Pública, consultada la Junta de Higiene, dictará las prescripciones del caso." Ley 39 de 1903 (26 de octubre) Sobre Instrucción Pública. Publicada por el Diario Oficial número 11,931 viernes 30 de octubre de 1903.

7. "Art. $8^{\circ}$ La inspección se ejerce no solamente sobre los maestros y alumnos, sino sobre todos los demás funcionarios que intervinieren en la Instrucción pública... Es un deber del inferior dar aviso a quien corresponda de la omisión o descuido del superior, para que se le haga efectiva la multa o responsabilidad en que haya incurrido..." Decreto número 491 de 1904 ( 3 de junio) por el cual se reglamenta la Ley 89 de 1903, sobre Instrucción Pública. Publicado por el Diario Oficial número 12,122 jueves 14 de julio de 1904. 


\section{correccional que se emplea, los progresos de la enseñanza, las dificultades y los medios para vencerlas ${ }^{8}$.}

Hasta aquí llama la atención la evidencia del sistema de vigilancia y control ejercido, es decir una forma de ejercicio del gobierno sobre los otros, puesta a funcionar; poder que mira, supervisa, juzga, examina buscando homogenizar unas formas de hacer en la escuela, tanto de maestros como de alumnos (denominación empelada en el documento original). Pero poder que también premia y corrige, este aspecto ya no en manos del Inspector sino del mismo maestro, quien es el encargado de poner a funcionar el sistema en el día a día de la escuela, a través de los reglamentos que se establezcan para tal fin ${ }^{9}$. Ejercicio disciplinar dirigido también a los cuerpos de los alumnos que se valió tanto de la moral como de la educación física, la gimnasia, la conducta, el aseo y el vestuario ${ }^{10}$.

8. "Art. 14. El Inspector local... hará un minucioso examen de la escuela, siguiendo las instrucciones que tengan del Inspector provincial. Se informará sobre las regulaciones y disciplina... su salubridad, las faltas cometidas, el carácter y conducta de los alumnos, el sistema correccional empleado y los efectos que haya surtido, los progresos de la enseñanza, la eficacia o inconvenientes de los sistemas empleados, las dificultades que se presenten... y los medios de vencerlas. Se hará presentar las listas de asistencia diaria, y examinará el mobiliario, los libros, los mapas y demás enseres..." Decreto número 491 de 1904 (3 de junio) por el cual se reglamenta la Ley 89 de 1903, sobre Instrucción Pública. Publicado por el Diario Oficial número 12,122 jueves 14 de julio de 1904.

9. "Capitulo XI. Del sistema correccional y de los premios. Art. 85. Los reglamentos establecerán el sistema de recompensas para premiar a los alumnos por su consagración, por su aprovechamiento y por su buen comportamiento... Para la distribución de los premios se cuidará de que haya la más estricta rigidez, de modo que no se prodiguen estos estímulos de honor, teniendo en cuenta... que no solamente deben premiarse la consagración y el aprovechamiento, sino también los esfuerzos del alumno para adquirir mérito moral. Art. 86. El moderno sistema disciplinario aconseja reducir a la menor expresión la necesidad de imponer castigos á los escolares;pero no debe tampoco prescindirse del deber de corregir al alumno. Solo cuando las palabras de consejo sean ineficaces por completo, será lícito imponer castigos más severos, proporcionados con la naturaleza de la falta cometida... Art. 101. Es deber de...

los Alcaldes poner á disposición de los maestros de escuela un Agente ó Comisario de Policía que anote los nombres de los niños que cometan faltas fuera de la escuela, para que le dé cuenta al Maestro. El mismo agente tiene la obligación de avisar en las casas... a fin de remediar la falta." Decreto número 491 de 1904 (3 de junio) por el cual se reglamenta la Ley 89 de 1903, sobre Instrucción Pública. Publicado por el Diario Oficial Número 12,122 jueves 14 de julio de 1904.

10. "Capitulo $\vee$ De la educación y de la enseñanza en general $1^{\circ}$ Educación Moral Art. 49...el Institutor trabajará por la cultura moral. La dignidad que imprimirá a todos sus actos y en su lenguaje, su respeto por la justicia, su afecto sincero por la infancia, le permitirán establecer una sabia disciplina, y le asegurarán la obediencia, el respeto y el amor de sus discípulos. Colocados así en las condiciones de un buen padre de familia, procurará que sus enseñanzas sean como las del hogar doméstico, llenas de sencillez, de bondad y de virtud. Para ello aprovechará todos los actos de la escuela, como las recreaciones, los juegos, los paseos, los mil incidentes de la vida escolar, para iluminar la conciencia de sus discípulos, a fin de desarrollar las buenas inclinaciones y de ejercitar y cultivar la voluntad en sus aplicaciones al bien... Art. 52. Los Institutores públicos tienen plena autoridad sobre los niños en todo lo que se refiera a su educación, y deben vigilar incesantemente su conducta, no solo dentro de la escuela sino fuera de ella, excepto dentro de los límites de la casa paterna. Cuidarán... de que los niños adquieran en sus maneras, palabras y acciones, hábitos de urbanidad, y los ejercitarán en la práctica de los deberes que el hombre bien educado tiene para con la sociedad en que vive... $4^{\circ}$ educación física Art. 60. La corrección en el vestido y un aseo riguroso son obligatorios para todos los niños. Los Institutores pueden rehusar la entrada a la clase a los alumnos que no reúnan estas condiciones, dando aviso por escrito a los padres respectivos... Art. 62. La calistenia y gimnasia, como parte indispensable de un sistema completo de educación se enseñarán en todas las escuelas, en las horas 
Es importante destacar además, el Articulo $1^{\circ}$ de la Ley 32 de 1936 "sobre igualdad de condiciones para el ingreso a los establecimientos de educación", el cual expresaba: "Ningún establecimiento de educación primaria, secundaria o profesional, podrá negarse a admitir alumnos por motivos de nacimiento ilegítimo, diferencias sociales, raciales o religiosas..." ${ }^{\prime 11}$ y el Artículo 3 en el que se pide: "Estimular el implantamiento de instituciones escolares de asistencia social, como restaurantes, cooperativas de nutrición, granjas, roperos, botiquines, etc...." del Decreto 1964 de 1938 que reorganiza la inspección Nacional de educación primaria. Se crearán y organizarán los orfanatos e internados de indígenas ${ }^{12}$, estos estarán a cargo de las Misiones Católicas.

Por otro lado, se hace visible, en el Decreto reglamentario de la Ley, lo que podría ser el nacimiento de una determinación de las carencias y desigualdades y del establecimiento -o institucionalización- de unas formas particulares también de concepción, enseñanza y gobierno sobre individuos que salen de un rango establecido. Al respecto llaman la atención aspectos como: la fundación del Instituto San Antonio en la ciudad de Bogotá para impartir enseñanza en agricultura, artes y oficios mecánicos a niños pobres ${ }^{13}$; o el Título I de la instrucción primaria que en su primer capítulo habla de la catequización de indígenas a quienes se refieren como tribus salvajes ${ }^{14}$; la división de las escuelas primarias entre rurales y urbanas o las escuelas de niñas y de varones ${ }^{15}$, así como también

destinadas a la recreación, según reglas sencillas y favorables al desarrollo de la salud y de las fuerzas de los niños..." Decreto número 491 de 1904 (3 de junio) por el cual se reglamenta la Ley 89 de 1903, sobre Instrucción Pública. Publicado por el Diario Oficial número 12,122 jueves 14 de julio de 1904 .

11. Artículo $1^{\circ}$ de la Ley 32 DE 1936 (febrero 20) "sobre igualdad de condiciones para el ingreso a los establecimientos de educación" Publicado en Diario Oficial número 23127 Bogotá, jueves 5 de marzo de 1936.

12. Artículo $1^{\circ}$ Ley 54 de 1931 .

13. "Art. 20. Autorizase al Gobierno para auxiliar la fundación y sostenimiento en Bogotá del Instituto de San Antonio, en el cual se dará enseñanza teórica y práctica de agricultura, y artes y oficios mecánicos, especialmente a niños pobres..." Ley 39 de 1903 (26 de octubre) Sobre Instrucción Pública. Publicada por el Diario Oficial número 11,931 viernes 30 de Octubre de 1903.

14. "Art. 29. El Gobierno tomará especial interés, de acuerdo con los respectivos Jefes de misiones, en atender a la evangelización e instrucción de las tribus salvajes." Decreto número 491 de 1904 (3 de junio) por el cual se reglamenta la Ley 89 de 1903, sobre Instrucción Pública. Publicado por el Diario Oficial número 12,122 jueves 14 de julio de 1904.

15. "Capítulo III. De las escuelas primarias. Art. 36. Las escuelas de enseñanza primaria se dividen en rurales y urbanas o de los distritos... Art. 41. Las escuelas de niñas serán regentadas por señoras de notoria respetabilidad y buena conducta. Las escuelas elementales de varones podrán ser confiadas a señoras que reúnan las aptitudes necesarias de instrucción y conducta virtuosa, siempre que a dichas escuelas no concurran niños mayores de doce años. En los Distritos en que no sea posible abrir escuelas de varones y de niñas separadamente, por falta de número, se establecerán a juicio del Inspector provincial, escuelas alternadas en los dos sexos, bajo la dirección de maestras de respetabilidad e idóneas." Decreto número 491 de 1904 (3 de junio) por el cual se reglamenta la Ley 89 de 1903, sobre Instrucción Pública. Publicado por el Diario Oficial número 12,122 jueves 14 de julio de 1904 . 
la diferenciación de algunas de las materias de enseñanza a razón del género al cual se pertenecía ${ }^{16}$.

Niños pobres, tribus salvajes distinciones entre lo rural y lo urbano o entre niñasy varonesson lasprimerasmanifestaciones dela determinación de carencias y desigualdades desde la política colombiana. Una forma de asumir de manera práctica la diferencia del lado de un pensamiento atomizado y antagónico. Formas de denominar a las que se sumarán otras, a partir de las pocas reformas que se harán en el transcurso de las décadas de los años 20, 30 y 40, encontrándose categorías como: Sociedad de Niños Desamparados, Asilo de Niños Huérfanos, alumnos incorregibles y desmoralizados. Niños aplicados y desaplicados, vicios y malas inclinaciones. "El alcoholismo como la lepra de las clases obreras y causa principal del aumento de la miseria y de la criminalidad"17, niños desamparados, ciegos y sordomudos ${ }^{18}$.

\section{Segundo momento: se expande la diferencia -marginalidad, criminalidad, anormalidad, enfermedad-}

Gestado desde finales de la década de los años 40, fortalecido y desarrollado en las décadas de los años 50 y 6o, cobijando buena parte del 70 del siglo $X X$, este momento es coincidente con discursos de la tecnología educativa, la planeación curricular, la teoría de sistemas, la teoría sociológica del desarrollo y la planeación estratégica. Es un período de postguerra que tiene que ver, por lo menos en un primer momento, con: la ola reformista de políticas educativas en Latinoamérica; la creación y fortalecimiento de los sistemas educativos en la región; la creación de los organismos de cooperación internacional y con lo que el profesor Alberto Martínez Boom denominará la expansión vertical y horizontal del sistema educativo y la escuela colombiana.

El proceso de escolarización se dio... en un doble sentido: horizontal y vertical... la escuela creció en sentido horizontal en

16. "Art. 66. En las escuelas alternadas habrá solo cuatro materias de enseñanza para los varones, y serán: Lectura, Escritura, Religión, y Aritmética. Lo mismo para las niñas, y, además, costura... Para las niñas serán alternadas la costura y la Aritmética." Decreto número 491 de 1904 (3 de junio) por el cual se reglamenta la Ley 89 de 1903, sobre Instrucción Pública. Publicado por el Diario Oficial número 12,122 jueves 14 de julio de 1904.

17. Art. 23 de la Ley 86 de 1928.

18. Decreto 1790 de 1930 que reglamenta algunos artículos de la Ley 56 de 1927. 
la medida en que amplió en forma significativa su cobertura... 1950 a 1970... multiplicó la población escolar (alumnos y maestros) ... también sus recursos financieros, su cubrimiento (urbano, rural), su equipamiento, etc.... también habría de llegar a las comunidades más remotas (marginados, indígenas). En sentido vertical, la escuela expansiva va a incorporar en su dinámica nuevas edades y niveles... preescolar... o la educación de adultos... y otros niveles más allá de los tradicionalmente conocidos...". (2004, p. 50)

Aquí el interés se concentrará en lo que se denominarán los sectores marginados o excluidos, que antes no formaban parte del sistema educativo, haciendo que éste se diversifique con la multiplicación de modalidades de atención. Estas alternativas recién creadas requerían de saberes y profesiones $y / 0$ funcionarios particulares que se hicieran cargo -sobre todo a finales de la década del 60 y buena parte del 70-, en ese sentido "En cada uno de los sectores a los que llegó, y en cada necesidad nueva que construyó, instaló un saber, una profesión y un tipo de funcionario... etnoeducación... educación comunitaria... educación preescolar... educación especial..." (Martínez Boom, 2004, p. 53). Se podría decir que es el segundo momento de institucionalización de la educación de anormales en Colombia. Se da una atención especializada en instituciones exclusivas para cada tipo de población.

Período en el que sigue vigente el enunciado de modernización, pero esta vez no con la idea de alcanzarlo a partir de la higienización, limpieza y pureza de la raza, sino con la función de convencer a los ciudadanos de su estado de subdesarrollo y de lo necesario de su participación para salir de él. Es un ejercicio de gobierno que, a partir de un proyecto integrador controlará y administrará grupos de población, grupos que serán potenciados, desde sus individualidades, en el trabajo y esfuerzo que requiere la modernización social y lo necesario de ella para salir del subdesarrollo.

En ese sentido, la respuesta ideal para los procesos de integración que se proponían de poblaciones antes socialmente excluidas (negros, indígenas, campesinos, refugiados, mujeres, menores de edad, anormales, pobres) fue la expansión educativa, a partir de cierto tipo de políticas que tendrán sustento en ideales y discursos democráticos, de convivencia pacífica y de cooperación internacional (Martínez Boom, 2004, pp. 49-50). Es justamente esta última -la cooperación 
internacional-, en cabeza de la Unesco y desde la década de los años 50, la que buscaría que los países miembros hicieran adaptación de sus sistemas educativos a las exigencias y postulados de la planificación. Para llegar a estos niveles de transformación y adecuación era necesario llevar a cabo estudios que dieran cuenta del estado actual, necesidades y requerimientos de cada país. Estos estudios contarían con la asesoría y acompañamiento de expertos.

A partir de esos estudios, en cuanto a políticas educativas, puede decirse que fue un periodo de mucho revuelo y cambios en la región que implicaron -para Colombia-, entre otras las siguientes acciones: mirada y reorganización de la educación como sistema; nacionalización de la enseñanza primaria; reformas al plan de estudios del bachillerato; reestructuración del Ministerio de educación; reformas a la educación superior; creación de una Oficina Nacional de Planeación, del Banco Educativo y de la Sección de Radio y Televisión del Ministerio de Educación; fundación de un Consejo Nacional de Televisión y una Junta Nacional de Censura; creación de un Consejo Nacional de Protección Infantil y de los institutos INSOR -para sordos- e INCI -para ciegos-; además de la solicitud de varias misiones que estudiarían temas diversos, entre ellos el educativo.

Entre las misiones más importantes, que dejaron recomendaciones para el sector educativo, se cuentan: la del Banco Internacional de Reconstrucción y Fomento (Misión Currie) dirigida por el economista Lauchlin Currie, realizada desde mediados de 1949 hasta 1950 y la Misión "Economía y Humanismo" (Misión Lebret) dirigida por el padre Louis Joseph Lebret, desarrollada desde finales 1954 hasta mediados de 1956. Ellas, a partir de los estudios realizados, los informes entregados y las recomendaciones sugeridas, terminaron teniendo efecto en la política educativa del país. Se evidencian estos efectos con mayor fuerza en el Informe del Proyecto para el I Plan Quinquenal publicado en septiembre de 1958 que dice: "... todos los estudios técnicos que se realizaron por la Oficina de Planeación de la Presidencia de la República o por misiones de Expertos internacionales, han contribuido a la fundamentación y respaldo de este I Plan Quinquenal..." (MEN, 1958, p. 3).

En la sección de política educativa, que posibilita continuar con el rastreo a la noción de diferencia, a la institucionalización de la educación para la misma en el país y a las disposiciones tomadas para llevarla a cabo, se pueden destacar varios documentos. A continuación, se 
detallarán algunos aspectos de estos que permiten evidenciar cómo la estrategia expansiva de la educación terminó creando nuevas categorías de población y formas de integración de las mismas en el recién reestructurado sistema de educación nacional.

Desde la Ley 83 de 1946 empiezan a visibilizarse algunas maneras particulares de asumir al otro, sobre todo al menor, como: menor infractor, en estado de abandono o de peligro moral o físico como aquel que requerirá ser sometido a medidas de asistencia y protección ${ }^{19}$. Disposiciones que contemplaban ser examinados por un médico psiquiatra, estudio a través del cual se determinaría si el menor debía ser internado en "casas de observación", enviado a un "hospital" o a un "centro de protección"20. Las casas de observación no tenían por función la corrección del menor, sino su estudio y se distinguirían de las "escuelas-hogares", de las "escuelas de trabajo" y de los "reformatorios", de allí debía resultar una determinación sobre el "tratamiento médicopedagógico" más conveniente ${ }^{21}$. Dicho tratamiento bien podía consistir en entregar al menor a una "institución idónea" que lo educara, internarlo en una "escuela de trabajo" o en un "reformatorio especial" o en una "granja agrícola" hasta su total reeducación ${ }^{22}$.

Igualmente se detiene la citada Ley en definir cada una de las categorías empleadas determinando:

19. "ARTíCULO 1o. El menor de diez y ocho años, hombre o mujer, que cometa alguna infracción penal, o que se halle en estado de abandono o de peligro moral o físico, será sometido a las medidas de asistencia, y protección preceptuadas en esta Ley". Ley 83 de 1946. Publicada en Diario Oficial No. 26.363, de 24 de febrero de 1947. (Resaltado en cursivasde la autora)

20. "ARTíCULO 5o. El personal del Juzgado de Menores será el siguiente: a) Un Médico Psiquiatra... f) Dos Delegados de Estudio y Vigilancia... ARTíCULO 6o. Son funciones del Médico Psiquiatra: estudiar los menores que le fueren enviados por el Juez, en estudio médico general y psiquiátrico, y proponer al Juez las medidas que estime más convenientes para la salud mental y física de tales menores, como el internamiento en la casa de observación, el envío a un hospital o centro de protección, la separación de la familia, etc., y hacer a las personas de la familia de los menores las visitas médicas...". Ley 83 de 1946. Publicada en Diario Oficial No. 26.363, de 24 de febrero de 1947. (Resaltado en cursivasde la autora)

21. "ARTíCULO 20. Cada Juzgado de Menores dispondrá de una casa de observación, cuya finalidad no es corregir al niño sino estudiarlo, que funcionará independientemente de las escuelas-hogares, escuelas de trabajo o reformatorios especiales. En la casa de observación, y por un término máximo de noventa días, se estudiará al menor integralmente en sus aspectos fisiológico, mental y moral y en sus reacciones individuales y sociales, y se consignarán las observaciones en una ficha que habrá de terminar con un dictamen sobre el tratamiento médico-pedagógico que deba aplicarse al menor...". Ley 83 de 1946. Publicada en Diario Oficial No. 26.363, de 24 de febrero de 1947. (Resaltado en cursivasde la autora)

22. "ARTíCULO 35. El fallo del Juez de Menores puede consistir en las siguientes medidas: $4^{a}$ Entrega del menor a una persona o Institución idónea, a fin de lograr su educación, bajo condiciones. $5^{a}$ Internamiento del menor en una escuela de trabajo, pública o privada, o en una granja agrícola especial para menores, pública o privada; y $7^{a}$ Internamiento del menor en un reformatorio especial para menores, por tiempo indeterminado, hasta cuando se obtenga la reeducación del menor o la formación de su sentido moral." Ley 83 de 1946. Publicada en Diario Oficial No. 26.363, de 24 de febrero de 1947. (Resaltado en cursivasde la autora) 
"... que un menor se halla en estado de abandono físico cuando carece de las personas que según la ley deban suministrarle alimentos, o cuando existiendo éstas, no tengan capacidad para suministrárselos... Un menor se halla en estado de abandono total cuando sus padres o las personas de quienes el menor depende lo incitan a la ejecución de actos perjudiciales para su salud física o moral; cuando se dedica a la mendicidad o a la vagancia, o frecuenta el trato con gente viciosa o de mal vivir, o vive en casas destinadas al vicio; y cuando ejerce algún oficio que lo mantiene permanentemente en la calle o en lugares públicos, o que pone en peligro su salud física o moral... Un menor se halla en estado de peligro físico o moral cuando las personas con quienes vive padecen de grave enfermedad contagiosa, o cuando le brindan de manera habitual malos ejemplos." ${ }^{23}$

Y de las instituciones de atención especializada que crea dice:

"Las escuelas-hogares son establecimientos de reeducación en que un pequeño número de corrigendos se confía a la dirección de un matrimonio experto en educación de anormales de carácter y en donde prima un régimen estrictamente familiar... Las escuelas de trabajo y las granjas agrícolas especiales para menores son establecimientos de reeducación en donde los menores destinados a ellas por los Jueces de Menores, están sometidos a un tratamiento de reforma, con una orientación hacia las industrias, o hacia la agricultura y la ganadería... Los reformatorios especiales para menores son establecimientos de reeducación a donde el Juez de Menores envía a los menores de notoria peligrosidad, o que se muestren díscolos a los medios ordinarios de reforma. En tales establecimientos se buscará de preferencia el orientar moralmente a los menores y el suministrarles una ocupación adecuada para la vida." ${ }^{24}$

Así mismo queda estipulado que los profesores y vigilantes en dichas instituciones serían maestros titulados y preferiblemente con estudios sobre educación de anormales ${ }^{25}$. En estos establecimientos los menores

23. Artículos 41,42 y 43 de la Ley 83 de 1946. Publicada en Diario Oficial No. 26.363, de 24 de febrero de 1947.

24. Artículos 55, 56 y 57 de la Ley 83 de 1946. Publicada en Diario Oficial No. 26.363, de 24 de febrero de 1947. (Resaltado en cursivas de la autora)

25. Artículos 62 de la Ley 83 de 1946. Publicada en Diario Oficial No. 26.363, de 24 de febrero de 1947. 
deberán ser clasificados y separados de acuerdo a su edad "mental y moral"26. Igualmente trata la Ley de la creación del Consejo Nacional de Protección Infantil el cual contará entre sus miembros con especialistas en derecho, sociología, cuestiones médico-sociales de la infancia, medicina pediátrica y en ciencias de la educación, pero sobre todo en educación de "díscolos y anormales"; dicho Consejo tendrá a su cargo varias funciones entre las que se contaban la asistencia al niño enfermo y anormal ${ }^{27}$; así como facilitar la apertura de instituciones de educación para dichos menores.

Un segundo documento es el Decreto 3157 de 1968 por el cual se reorganiza el Ministerio de Educación Nacional y se estructura el sector educativo de la Nación, es el momento de creación de la División de Educación Especial como parte estructural de este organismo; en el Artículo $4^{\circ}$ puntualiza en uno de sus apartados: "... 3. División de Educación Especial. a) Sección de Educación para subnormales. b) Sección de Educación para Sobresalientes...", delimitando sus funciones en el Artículo 19 así: "Corresponde a la División de Educación Especial a través de sus secciones, promover programas y servicio de educación especial, tanto para alumnos subnormales, como para estudiantes sobresalientes." 28

Por otra parte, en el I Plan Quinquenal de Educación, en la sección 1.7 correspondiente a los aspectos sociales de la educación se dice:

"En todo programa de salud escolar no se puede pasar por alto la situación de miles de niños defectuosos o incapacitados

26. Artículos 63 de la Ley 83 de 1946. Publicada en Diario Oficial No. 26.363, de 24 de febrero de 1947.

27. "ARTíCULO 97. Créase el Consejo Nacional de Protección Infantil, integrado por cinco miembros, elegidos para un periodo de tres años, así: uno nombrado por el Presidente de la República, que deberá ser abogado especializado en ciencias penales; uno nombrado por el Arzobispo Primado, que deberá ser sacerdote experto en sociología; uno por el Comité Nacional de la Cruz Roja, que deberá ser muy versado en cuestiones médico-sociales de la infancia; uno nombrado por la Sociedad Colombiana de Pediatría, que deberá ser médico pediatra; y el quinto, por el Juez de Menores de Bogotá, que deberá ser especializado en ciencias de la educación y en educación de díscolos y anormales. ARTÍCULO 99. El Consejo, sea por medio de los Comités municipales, tendrá a su cargo todo lo referente a la prestación y organización de los servicios sociales... e) Asistencia al niño anormal y enfermo... g) Asistencia y protección hasta la mayor edad, de los niños abandonados o en peligro; $h$ ) Asistencia y protección a los niños infractores de las normas penales; i) Asistencia y protección a la madre y al niño que trabajan... 2 $2^{a}$ Aunar todas las iniciativas de entidades públicas o privadas encargadas de la protección del niño y de la madre, a fin de que se obtengan la protección y asistencia del niño desnutrido, enfermo, abandonado, en peligro, o delincuente; 11 . Procurar la fundación de establecimientos de educación para menores abandonados, delincuentes y anormales..." Ley 83 de 1946. Publicada en Diario Oficial No. 26.363, de 24 de febrero de 1947.

28. Decreto 3157 de 1968 por el cual se reorganiza el Ministerio de Educación Nacional y se estructura el sector educativo de la Nación. Publicado en Diario Oficial 32697 miércoles 29 de enero de 1969 
entre los que podemos considerar: niños tullidos por lesiones de cerebro, fiebre reumática, etc. Ciegos, sordos, asmáticos, diabéticos, epilépticos, niños de cociente de intelectualidad normal y retardados mentales o con deficiencias debidas al ambiente. Este grupo de niños también tiene derecho a una educación apropiada. Los menores pueden obtener educación especial en el hogar; otros pueden incorporarse a un grupo de niños normales, siempre que sus defectos sean leves o sean corregidos, como el caso de la sordera que mejora con aparatos especiales, pero hay un grupo que necesita de una escuela especial para educarlos lo más adecuada y sanamente posible dentro de sus limitaciones mentales y físicas. Muchos individuos de $\mathrm{Cl}$ bajo tiene habilidades manuales que, una vez descubiertas, pueden ser encauzadas; individuos defectuosos físicamente pueden tener habilidades artísticas y la Nación tiene la responsabilidad de convertirlos en ciudadanos útiles a sí mismos, creándoles un modo de aceptación y comprensión hacia sus deficiencias y de máxima utilización de sus propias habilidades. El establecimiento de escuelas especiales para niños, incapacitados física y mentalmente, es una necesidad y debe formar parte de cualquier programa de desarrollo educacional en un país." 29

En relación con lo tratado en el documento anterior se encuentra el Decreto 1038 de 1974, por el cual se establecen unos cursos de capacitación ${ }^{30}$, en la procura de prevenir enfermedades mentales, en este Decreto se enfatiza, entre otros, en los siguientes aspectos:

"...en la utilización del maestro como promotor de salud mental... la etapa preescolar requiere especial cuidado tanto desde el punto de vista educativo general... la prevención de los trastornos que pueden afectar la salud mental... los altos índices de repitencia escolar en el país se deben, al menos en parte, a dificultades para el aprendizaje... los niños con problemas o limitaciones en el aprendizaje necesitan tratamiento adecuado para sus situaciones específicas y para el mantenimiento de la salud mental... son susceptibles a trastornos psicológicos que puedan conducir a la vagancia,

29. Ministerio de Educación Nacional. Oficina de Planeación. Informe del Proyecto para el I Plan Quinquenal. Tomo I. Bogotá. Junio de 1957. Págs. 27 y 28. (Resaltado en cursivas de la autora)

30. Decreto 1038 de 1974, por el cual se establecen unos cursos de capacitación. Publicado en Diario Oficial 34110 viernes 28 de junio de 1974. 
la prostitución, las farmacodependencias, la delincuencia juvenil, etc...." ${ }^{\prime 1}$

Por otro lado, en el Decreto 2499 de 1973

"...se reconocen la educación preescolar y la educación especial como niveles educativos sujetos a la aprobación y vigilancia del Ministerio de Educación... los niveles preescolar y de educación especial exigen maestros idóneos en sus diversas modalidades... es indispensable que educadores, consejeros escolares, psicológicos, psicopedagogos, trabajadores sociales, sociólogos, antropólogos y otros profesionales vinculados a la educación, adquieran o mejoren los conocimientos necesarios para promover la salud mental... en consecuencia, todos los profesionales de que trata el considerando anterior pueden especializarse o perfeccionar sus estudios sobre un programa básico común... debido a la limitación de recursos económicos del país conviene aprovechar al máximo el personal que está en servicio activo en la educación facilitándole los medios para que perfeccione sus conocimientos o para que adquiera una especialización..." ${ }^{12}$

Otro hecho que llama la atención es la cantidad de Decretos que se empiezan a encontrar trabajando en torno a la reestructuración y reorganización del Ministerio de Educación Nacional y el lugar que va ocupando en él la modalidad de Educación Especial, por ejemplo para el año 1976 está el Decreto 088 de $1976^{33}$ en el Art. $5^{\circ}$ y en el parágrafo que lo acompaña, especifica:

"Se llamará Educación Especial la que se refiera a los sobresalientes y a quienes presenten deficiencias físicas, mentales, emocionales, sociales o tengan dificultades especiales en el aprendizaje. Parágrafo. La Educación Especial estará integrada a la Educación Formal o No Formal. El Gobierno establecerá programas adecuados, estimulará la iniciativa

31. Decreto 1038 de 1974, por el cual se establecen unos cursos de capacitación. Publicado en Diario Oficial 34110 viernes 28 de junio de 1974.

32. Decreto 2499 de 1973 , por el cual se establecen unos cursos de capacitación. Publicado en Diario Oficial 34110 viernes 28 de junio de 1974. (Resaltado en cursivas de la autora)

33. Decreto 088 de 1976 por el cual se reestructura el sistema educativo y se reorganiza el Ministerio de Educación Nacional. Publicado en Diario Oficial 34495 lunes de febrero de 1976 
privada y promoverá la formación de personal docente en este ramo y la investigación en el Educación Especial."34

Más adelante, en el Art. 11 habla de los programas regulares para la educación de las comunidades indígenas y de cómo estos tendrán en cuenta la realidad antropológica de los grupos, fomentando la conservación y la divulgación de sus culturas autóctonas. En el Art. 15 detallará la constitución del MEN, específicamente cita los institutos INCl -para ciegos- e INSOR -para sordos- como establecimientos públicos adscritos al Ministerio; por otra parte, dentro de la estructura administrativa del mismo (Art. 17) se contempla la División de Educación Especial adscrita a la Dirección general de Administración e inspección educativa a la que se le asignan, entre otras, las siguientes funciones:

"... i. Preparar, en coordinación con la Dirección General de Capacitación y Perfeccionamiento Docente, Currículo Medios Educativos, las normas reglamentarias sobre la administración y la inspección de la Educación Especial y la Educación No Formal... k) Cooperar con la Dirección de Capacitación y Perfeccionamiento Docente, Currículo y Medios Educativos en la capacitación y perfeccionamiento del personal docente para los programas de Educación Preescolar, Educación, Educación Especial y Educación No Formal..."35

De acuerdo a lo tratado en el Art. 11 del citado decreto, vale la pena ampliar que los procesos de formulación de políticas educativas dirigidas a los pueblos indígenas se iniciaron a finales de la década del 70 con la promulgación de los decretos o88 de 1976 y 1142 de 1978, con los cuales se reestructuró el Sistema Educativo y el Ministerio de Educación Nacional. Dentro de los cambios que se introdujeron, se establecieron: la participación, la cooperación, la investigación, el uso de lenguas nativas, la gratuidad, la financiación descentralizada, la elección especial para etnoeducadores y los calendarios flexibles.

Sin lugar a dudas es este un periodo en el que se amplían significativamente las miradas en torno a la anormalidad, siempre con mayor concentración en la infancia, que será el grupo de población hacia

34. Decreto 088 de 1976 por el cual se reestructura el sistema educativo y se reorganiza el Ministerio de Educación Nacional. Publicado en Diario Oficial 34495 lunes de febrero de 1976. (Resaltado en negrilla de la autora)

35. Decreto 088 de 1976 por el cual se reestructura el sistema educativo y se reorganiza el Ministerio de Educación Nacional. Publicado en Diario Oficial 34495 lunes de febrero de 1976. 
el cual se apuntalen la mayoría de disposiciones de la política educativa en su afán por ampliar y extender la cobertura de su servicio. Entre las categorías que vendrán a sumarse para este mapeo se encuentran: menor infractor; menor en estado de abandono; menor en estado de peligro moral o físico; corrigendos; anormales de carácter; menores de notoria peligrosidad; díscolos niño enfermo; niño anormal; alumnos subnormales; estudiantes Sobresalientes; niños defectuosos; niños incapacitados; niños tullidos; ciegos, sordos, asmáticos, diabéticos, epilépticos; niños de cociente de intelectualidad normal; niños retardados mentales; niños con deficiencias debidas al ambiente; individuos defectuosos físicamente; niños incapacitados física y mentalmente; niños con dificultades de aprendizaje.

Pero también debe destacarse el interés que empiezan a despertar otras poblaciones como los grupos indígenas, a quienes se dirigen normas especiales para poder ser involucrados en los planes de acción del Ministerio de Educación y en las prácticas del Sistema Educativo Nacional. Así mismo se ven, de manera más concreta, algunas acciones para la formación de docentes especializados y se empieza a hablar de Educación Especial.

Afinamiento de unas acciones de gobierno sobre un grupo de población que ya no se dedicará al esfuerzo por corregir, sanar y homogenizar sino que integrará, hará visible para continuar con un ejercicio de técnicas correctivas hacia los cuerpos, pero que empezará a combinar estrategias más amplias de examen, vigilancia, clasificación, separación, en las que vinculará a los maestros, padres de familia y expertos en varios saberes que empiezan a hacerse necesarios para el tratamiento médicoeducativo de estos grupos poblacionales. Ejercicio de administración de la población, control que detectará y potenciará las habilidades que cada individuo tenga.

\section{Tercer momento: el giro o descentramiento de la mirada. De las necesidades educativas especiales y la integración, a la inclusión de poblaciones vulnerables.}

Los últimos años de la década del 70 y las dos últimas décadas del siglo $X X$, tienen que ver con: el fortalecimiento (en un primer momento) y mayor intervención de los organismos de cooperación internacional 
(en el resto del periodo); un alto nivel de endeudamiento de los países latinoamericanos a raíz de las fuertes inversiones para el despliegue de la estrategia de expansión de la educación; un concentrado interés por lo económico y financiero; la idea de lo ineficaz del modelo de la oferta y lo necesario de un modelo distinto -la demanda, el mercado-. Este momento coincide con una apertura hacia las intercomunicaciones, la globalización, el fin de las fronteras, la mundialización de las políticas; un mundo que hablará del respeto a la diferencia, mundo plural en el que empieza a cobrar importancia el discurso de la inclusión y la multiculturalidad.

Se podría decir que es un período en el que continua vigente el enunciado de modernización; una modernización no civilizadora pero en busca del progreso a través de la modernización de los sistemas educativos, la introducción de nuevas tecnologías, el manejo de las comunicaciones y la priorización en la planificación, sobre todo en el uso de los recursos (la eficiencia y la eficacia); una modernización que ya no deja todo al Estado sino que empieza a hacer ciertos niveles de apertura hacia recursos privados.

El mundo empieza a experimentar una nueva fase del capitalismo, así como formas distintas del ejercicio del gobierno de los países, fenómenos que vienen a surtir efectos en distintos órdenes, de acuerdo a sus formas de apropiación y puesta en funcionamiento. En ese sentido, en particular para Latinoamérica, se tendría que hablar de la apropiación de las ideas planteadas por el neoliberalismo norteamericano en las formas de gubernamentalidad de los países y de la globalización como uno de los efectos de la actualidad del capitalismo. Por otra parte, están las formas distintas de ejercicio del gobierno en los países que evidencian la gubernamentalización de la sociedad a través, básicamente, de la racionalización del ejercicio de poder.

Retomando algunos análisis de Michel Foucault, desarrollados en $L a$ Gubernamentalidad, se podría afirmar que se estaría en un momento de gobierno denominado el estado de gobierno o de economía política, que como toda forma de ejercicio de gobierno tiene su propia lógica del poder, su propio funcionamiento y algunas modificaciones. En particular, esta forma de gobierno está caracterizada por

"La introducción de la economía dentro del ejercicio político: eso es... lo que constituirá el desafío esencial del gobierno... 


\begin{abstract}
Gobernar un Estado será... poner en práctica la economía, una economía de todo el Estado... tener con respecto a los habitantes, a las riquezas, a las conductas de todos y cada uno, una forma de vigilancia, de control... el arte de gobernar es precisamente el arte de ejercer el poder en la forma y según el modelo de la economía... la esencia misma de este gobierno... va a tener como objeto principal lo que ahora llamamos economía... En eso consiste, por tanto, lo que es gobernar y ser gobernado" (Foucault, 1999, p. 179)
\end{abstract}

En ese sentido, la globalización vendría a ser uno de los efectos de la gubernamentalidad liberal y de la nueva fase que está viviendo el capitalismo en el mundo. Esta traerá, efectos y modos de ser de los sujetos particulares, llegando incluso a convertirse en una forma de vivir. Algunos de estos efectos, tienen énfasis en las nuevas formas de la política, particularmente en las políticas educativas.

En efecto, hay una reactivación del discurso de los derechos humanos; también se presencia en la década de los go que varios países de Iberoamérica hacen revisión y modificación de sus constituciones y a partir de allí se generan una serie de transformaciones y nuevas políticas que abarcan desde las áreas de la salud, la economía, el trabajo y la educación.

En la década del 70 -con el informe Warnok en 1978- y buena parte de la del 80 los discursos de la integración, las necesidades educativas y las adaptaciones curriculares, serán temas de interés en una parte de los sistemas educativos y se dará continuidad a la atención en instituciones exclusivas, pero ahora integrando todo tipo de población -con discapacidades distintas-. Para la década del go estos intereses se concentrarán en los enunciados de la educación para todos -Jomtiem en 1990-, la integración y las necesidades educativas especiales -Salamanca en 1994-; quedando planteada, además -desde el Informe Delors en 1996- la apertura a un mundo multicultural, que abogue por el respeto a las diferencias y en donde vivamos todos juntos.

Por su parte, la integración se basó, y continúa sustentándose, en unos principios y fundamentos que buscan básicamente la normalización, esto es, el acercamiento de la vida a una condiciones lo más normales posibles; con una concentración fuerte en los derechos humanos; en el sujeto -desde sus dificultades-, en cómo él logra integrarse al medio, 
cómo normaliza los comportamientos y actitudes para integrarse y socializarse. Práctica educativa y de formación de sujetos en la que se combinan formas anatomo-políticas y biopoliticas del ejercicio del biopoder. Integración, adaptaciones curriculares, educación para todos, necesidades educativas especiales, empezarán a constituirse en las condiciones que darán posibilidad a discursos de la inclusión como los que se escuchan hoy.

Por otro lado, la inclusión tiene mayor concentración en los medios, en el ambiente, en los apoyos que este provee y no tanto en el sujeto. Se habla ahora de orientaciones educativas para poblaciones vulnerables; es un discurso con sustento en el despliegue de la concepción de desarrollo humano, que se visibiliza en la implantación de políticas de inclusión social y en una reactivación de la perspectiva de los derechos humanos; idea que ha logrado establecer relación con los diversos niveles de formación, inclusive con los que se han denominado formación para el trabajo o formación en competencias laborales.

Ahora bien, las principales ideas y proyecciones de trabajo en el sector educativo para con las personas que difieren de los estándares de normalidad de la mayoría, que en adelante tendrán los países Iberoamericanos y en particular Colombia, tienen que ver con cuatro documentos internacionales que durante este período sentarán las bases para hablar de integración, inclusión y que posteriormente, de acuerdo a sus grados y tipos de apropiación, tendrán efectos en gran parte de la política educativa. Dichos documentos son:

- El Informe Warnock (1978), presentado por la Comisión de Investigación sobre la Educación de Niños y Discapacitados Jóvenes en Londres, a la cabeza de Helen Mary Warnock (miembro de la Academia Británica, filósofa británica especializada en filosofía moral, filosofía de la educación y filosofía de la mente, estudiosa del existencialismo) y titulado Necesidades Especiales; presentaba los resultados de una vasta investigación Ilevada a cabo en Inglaterra, Escocia y Gales principalmente-, pero que extendió sus indagaciones y visitas hasta Estados Unidos de América, Canadá, Dinamarca, Suecia, Holanda y Alemania Occidental; esta exploración buscaba fundamentalmente llegar a la formulación de una serie de recomendaciones para el desarrollo futuro de la educación especial, entre las que se pueden destacar las siguientes: 


\begin{abstract}
El servicio de la educación especial será una modalidad que en algún momento de la vida escolar va a requerir cualquier niño; eliminar las categorizaciones, clasificaciones, agrupaciones o separaciones de los estudiantes con alguna discapacidad; el término "niños con dificultades de aprendizaje" debe tener un uso más amplio designando con él tanto a niños del sistema regular como a los que sean atendidos por la educación especial; las escuelas especiales o centros de educación especial deben continuar funcionando para la atención de menores con discapacidades complejas; los demás niños deben ser integrados a la escuela regular; incorporar un concepto más amplio que permita describir a un determinado grupo de niños que requieren de los servicios especiales como: aquellos con dificultades significativas en el aprendizaje, o con trastornos emocionales o de comportamiento, así como aquellos con discapacidades de la mente o el cuerpo.
\end{abstract}

- La Conferencia Mundial sobre Educación para Todos: Satisfacción de las Necesidades Básicas de Aprendizaje - Jomtien, Tailandia - 1990. Este documento reconoce como de vital importancia la educación básica para el progreso social, constituyéndose en una guía útil para los gobiernos, las organizaciones internacionales, los educadores y los profesionales del desarrollo cuando se trata de elaborar y de poner en práctica políticas y estrategias destinadas a perfeccionar los servicios de educación básica y establece un lugar de gran importancia para la educación en la política de desarrollo humano. Sus efectos se han hecho sentir en acciones como: universalización de la enseñanza primaria, la erradicación del analfabetismo en adultos, el mejoramiento de la calidad en la educación básica y a poner en funcionamiento medios más eficaces y baratos para satisfacer las necesidades básicas de aprendizaje de diversos grupos desasistidos. Es tal vez esta última acción la que más ha servido para catapultar la modalidad de atención en integración para poblaciones con algún tipo de discapacidad y se evidencia en el documento que a continuación se detalla.

- La Conferencia Mundial sobre Necesidades Educativas Especiales: Acceso y Calidad - Salamanca, España, 1994. A través de este evento y del documento que resultó del mismo se buscaba básicamente promover el objetivo de la Educación para Todos, a través del examen de la política en función de la educación integradora, la capacitación a escuelas para la atención a todos los niños, sobre todo a los que tienen necesidades 
educativas especiales. Se encuentran en este documento enunciados importantes que se constituirán en el piso de posibilidad para lo que después se empezarán a denominar las políticas de inclusión:

"Estos documentos están inspirados por el principio de integración y por el reconocimiento de la necesidad de actuar con miras a conseguir "escuelas para todos" esto es, instituciones que incluyan a todo el mundo, celebren las diferencias, respalden el aprendizaje y respondan a las necesidades de cada cual. Como tales, constituyen una importante contribución al programa para lograr la Educación para Todos y dotar a las escuelas de más eficacia educativa. Las prestaciones educativas especiales - problema que afecta por igual a los países del norte y a los del sur - no pueden progresar aisladamente, sino que deben formar parte de una estrategia global de la educación $y$, desde luego, de nuevas políticas sociales y económicas. Requieren una reforma considerable de la escuela ordinaria. ${ }^{136}$

- Informe a la UNESCO de la Comisión Internacional sobre la Educación para el Siglo XXI. Presidido por Jacques Delors - 1996. Este documento, más amplio y general que el anterior, dentro de sus planteamientos deja entrever una forma de reactivación de los derechos humanos, pero pensados ahora en función de unos discursos de paz y justicia que han puesto como ideales las democracias más recientes en Latinoamérica, algunos de sus interesantes enunciados son:

"... la educación constituye un instrumento indispensable para que la humanidad pueda progresar hacia los ideales de paz, libertad y justicia social... función esencial de la educación en el desarrollo continuo de la persona y las sociedades... como una vía, ciertamente entre otras pero más que otras, al servicio de un desarrollo humano más armonioso, más genuino, para hacer retroceder la pobreza, la exclusión, las incomprensiones, las opresiones, las guerras, etc. ...La educación es también un clamor de amor por la infancia, por la juventud que tenemos que integrar en nuestras sociedades en el lugar que les corresponde, en el sistema educativo indudablemente, pero también en la familia, en la comunidad de base, en la nación..."37 (pp. 7-8)

36. Conferencia Mundial sobre Necesidades Educativas Especiales: Acceso y Calidad Salamanca, España, 1994. Págs. 3 y 4. (cursivas de la autora)

37. Informe a la Unesco de la Comisión Internacional sobre la Educación para el Siglo XXI. Presidido por Jacques Delors - 1996. (cursivas de la autora) 
Ahora bien, para el caso de la política educativa colombiana, puede decirse que la década del 70 y una parte de la del 80 no tuvieron mayor significación ni movimientos importantes, simplemente se expidieron normas dando continuidad al funcionamiento de algunas instituciones ya conformadas como el INCl${ }^{38}$ o reglamentando algunos apartados de leyes expedidas para grupos de población particulares, como es el caso de las comunidades indígenas ${ }^{39}$ o decretos con algunos requerimientos particulares a nivel curricular, como eximir de cursos de idiomas extranjeros a los estudiantes con necesidades educativas especiale $4^{40} \mathrm{O}$ con algunas modificaciones en cuanto al nombramiento de profesores para educación especial en sectores alejados y de difícil acceso en el país ${ }^{41}$ o con algunas reglamentaciones particulares en cuanto a la modificación en el cobro de las matrículas ${ }^{42}$.

Se destaca el Decreto 2230 de 1986, por el cual se crea el Comité Nacional de Lingüistica Aborigen que sería un organismo que, entre otras funciones, asesoraría al Gobierno Nacional en aspectos de investigación, formulación de políticas, enseñanza, difusión y uso y conservación de las lenguas indígenas y criollas ${ }^{43}$. Igualmente los Decretos 0428 y 1895 del mismo año por medio de los cuales, respectivamente, se establece el Plan de estudios para la educación básica de adultos y se reglamenta un artículo de la Ley 20 del 82 regulando un fondo especial del SENA para la capacitación de menores trabajadores, entre los que se incluyen aquellos que presenten algún tipo del incapacidad física o minusvalía ${ }^{44}$.

38. Decreto Número 0932 de 1977 (mayo 2) por el cual se modifican los Estatutos de Instituto Nacional para Ciegos. INCI Decreto Número 0577 de 1978 (abril 4) por el cual se modifican los estatutos del Instituto Nacional para Ciegos, INCI.

39. Decreto Número 1142 de 1978 (junio 19) por el cual se reglamenta el artículo 118 del Decreto - ley número 088 de 1976 sobre educación de las comunidades indígenas

40. Decreto Número 34 de 1980 (enero 11) por el cual se eximen de cursar idiomas extranjeros a alumnos de Educación Especial. Publicado en Diario Oficial 35449 lunes 4 de febrero de 1980 .

41. Decreto Número 85 de 1980 (enero 23) por el cual se introducen unas modificaciones al Decreto extraordinario 2277 de 1979. Publicado en: Diario Oficial miércoles 27 de febrero de 1980.

42. Decreto Número 3486 de 1981 (diciembre 9) por el cual se establece el régimen de matrículas y pensiones, becas, Juntas Reguladoras de Matrículas y Pensiones y otras variables de costos en los establecimientos educativos no oficiales de los niveles preescolar, básica (primaria y secundaria), media vocacional y educación especial, y se dictan otras disposiciones.

43. Decreto Número 2230 de 1986 (julio 14) por el cual se crea el Comité Nacional de Lingüística Aborigen.

44. Decreto Número 0428 de 1986 (febrero 7) Por el cual se establece el Plan de Estudios para la Educación Básica Primaria de Adultos y Decreto Número 1895 de 1986 (junio 13) por el cual se reglamenta el artículo 20 de la Ley 20 de 1982, que regula el Fondo Especial de Capacitación de los trabajadores menores de dieciocho (18) años de edad, por parte del Servicio Nacional de Aprendizaje, SENA. 
Será la década del go y con ella la Ley General de Educación o ley 115 de 1994 la que posibilitará reconocer varios aspectos que aportarán para el mapeo que se realiza aquí. Con la promulgación de la Constitución Política de 1991, la educación se reconoce como un derecho de las personas "y un servicio público que tiene una función social... que busca el acceso al conocimiento, a la ciencia, a la técnica y a los demás bienes y valores de la cultura" y que forma "en el respeto a los derechos humanos, a la paz y a la democracia; y en la práctica del trabajo y la recreación, para el mejoramiento cultural, científico, tecnológico y para la protección del ambiente" 45 .

De acuerdo a lo anterior, la Ley General de Educación Colombiana (Ley 115), en el Título III de Modalidades de Atención educativa a poblaciones, desarrolla cinco apartados dirigidos a la educación de: personas con limitaciones y capacidades excepcionales; adultos; grupos étnicos; campesinos y rehabilitación social. Respecto del primero dice que esta educación es para personas con limitaciones físicas, sensoriales, psíquicas, cognoscitivas, emocionales o con capacidades intelectuales excepcionales, pertenece al servicio público educativo y busca la integración académica y social de los educandos, para lo cual se pondrán a funcionar programas de apoyo pedagógico, a través de aulas especializadas ${ }^{46}$.

En cuando a la educación para adultos, especifica que es la ofrecida a personas en edad relativamente mayor a la aceptada en la educación formal, personas que desean suplir y complementar la formación o también a quienes deben validar sus estudios; esta modalidad se desarrollará a través de educación a distancia y semipresencial. Además se fomentará la educación no formal para este grupo de personas ${ }^{47}$. Por otro lado, sobre los grupos étnicos, dice que son grupos o comunidades que poseen lengua, cultura y tradiciones propias y autóctonas, en ese sentido, la etnoeducación, además de estar orientada por el objeto, principios y fines generales de la educación colombiana, debe incluir las particularidades sociales que caracterizan a dichos grupos. En Colombia se han identificado como grupos étnicos los pueblos indígenas, las comunidades negras, afrocolombianas, raizales del Archipiélago de San Andrés, Providencia y Santa Catalina y el pueblo Rom o Gitano ${ }^{48}$.

\footnotetext{
45. Constitución Política de 1991. Artículo 67.

46. Ley 115 de febrero 8 de 1994. Título III. Capítulo I. Arts. 46 - 49.

47. Ley 115 de febrero 8 de 1994. Título III. Capítulo I. Arts. 50 - 54.

48. Ley 115 de febrero 8 de 1994. Título III. Capítulo I. Arts. 55 - 63.
} 
La educación campesina y rural, por su parte, comprende las modalidades formal, no formal (ahora educación para el trabajo y desarrollo humano) e informal y comprenderá básicamente formación básica en actividades agropecuarias, pesqueras, forestales, etc. 49 Finalmente, en el apartado de educación para la rehabilitación social, esta es caracterizada como aquella que comprende programas educativos ofrecidos a personas que presentan comportamientos individuales y sociales que les exigen procesos educativos para reincorporarse a la sociedad..$^{\circ}$

Serán todas estas normativas, sus formas de concebir al otro, las alternativas de educación y formación que proponen y ponen a funcionar, los elementos que irán constituyéndose en las condiciones de posibilidad para lo que vendrá a ser el inicio de una campaña y despliegue de la estrategia de la inclusión social y educativa para poblaciones vulnerables. Es este momento el del giro, el de descentramiento de la mirada y cambio de interés, pero ello no quiere decir que hoy se estén viviendo en Colombia procesos de inclusión definitivos, totales, únicos. Al contrario, prácticas propias de la integración aun conviven y se disputan el terreno con la naciente idea de la inclusión. Podría decirse que estos dos momentos o formas de biopoder aún están presentes con prácticas antomo-políticas como biopolíticas.

\section{Reto y apuesta: la diferencia}

Con el trazado del mapa que se acabó de hacer, se muestran las distintas formas de visibilidad y de normatividad que ha tenido en Colombia la diferencia, por lo menos desde la política educativa en el siglo XX. Es un primer acercamiento que posibilita decir que las poblaciones que salen del rango de lo establecido como normal en cada período, han estado en la mira de la educación desde perspectivas que establecen sesgo y marginalidad; miradas y formas de decir que han pretendido situar a los sujetos en prácticas de reeducación que buscaban integrar lo anormal a la sociedad porque se necesitaba de ellos para cumplir estrategias de desarrollo, modernización, civilidad, progreso y productividad.

Formas de hacer, visibles en primera instancia en la escuela, pero que poco a poco se han ido extendiendo al grueso de las acciones

49. Ley 115 de febrero 8 de 1994. Título III. Capítulo I. Arts. $55-63$.

50. Ley 115 de febrero 8 de 1994. Título III. Capítulo I. Arts. 68 - 71. 
de la sociedad colombiana que no dan cuenta de una diferencia asumida como acontecimiento, pues se le continúa situando del lado de la representación, la analogía, la oposición, el antagonismo, la contradicción o la semejanza. No una diferencia como forma de pensamiento inmanente al sujeto -a cualquier sujeto-; sino leída como necesidad de completud.

Igualmente, este mapeo ha posibilitado mostrar las formas de institucionalización que se han venido configurando, en el medio colombiano, en torno de la educación de poblaciones diferenciales. Estas se conforman a partir de dos preguntas suscitadas por estos grupos de población: la primera por lo que se sabe acerca de ellos; la segunda, cuestiona acerca de lo que se puede hacer con ellos. La forma en que se han resuelto estas cuestiones da cuenta de la creación de una serie de saberes, profesiones, cargos, especialistas; pero además de categorías o formas de nombrar que varían de acuerdo a la gradación que socialmente se establece en cada momento de la anormalidad y por supuesto también de la concreción en una serie de instituciones especializadas en la atención de estos grupos y sus características muy particulares.

Es justamente desde esas formas (series) de institucionalización: saberes (profesiones), prácticas (instituciones) y sujetos (maneras de nombrar), que se integran unas relaciones de fuerza (formas de gubernamentalidad) y unos modos de educar y tratar a estos grupos de población. Esta institucionalización de un ejercicio de gobierno se convierte en un instrumento de poder que se encontrará con formas de despliegue del saber, integración que, en últimas terminará asignando un lugar, unas maneras de entender, ser y hacer particulares en torno a la diferencia. Unas veces ejercicio anatomo-político, pero en la mayoría de las oportunidades en convivencia lo anatomo-político con el ejercicio biopolítico.

Ahora bien, de acuerdo a lo antes dicho, si se logra asumir la diferencia desde relaciones diferenciales, necesariamente en el ámbito educativo se podrán lograr afecciones que aumenten la potencia de obrar y comprender. Obrar y comprender desde cada uno; obrar y comprender desde los grupos. No es tanto la necesidad de sensibilización ante la diferencia, ni del respeto a las diferencias, sino del obrar y el comprender; de lo que se hace, de lo que se sabe, de la forma como se mire y se asuma esa diferencia. 
Es necesario hacer efectivo lo que ha venido quedándose en el papel: el reconocimiento a la diferencia. El enunciado está planteado, el acuerdo en torno a su cumplimiento no tiene discusión, pero el siguiente paso es hacerlo efectivo en las prácticas: desde la elaboración de políticas educativas hasta en el día a día de los procesos educativos, para que en la generalidad -o en el afán de homogenizar- no termine perdiéndose la pluralidad.

Se hace preciso que las escuelas sigan replanteando algunas de sus prácticas, una mirada hacia dentro para graduar la inflexibilidad de algunos procesos llevados a cabo en las instituciones escolares posibilitaría la educación plural. Escuelas que den respuestas diferenciadas en cuanto a adecuaciones y pertinencia del currículo; en lo referente a la evaluación que, en lugar de ser generalizada, logre adecuarse a los estudiantes: una escuela con programas de refuerzo y apoyo; pensar las estrategias pedagógicas para no caer en prácticas repetitivas, que no avancen, pero que tampoco promuevan automáticamente, por conveniencia, por pesar o por obligatoriedad de una norma.

Tal vez es este el momento, ya hay condiciones de posibilidad para que se pueda hablar de una educación para la diferencia y en la diferencia: ya se han escrito, defendido y promovido documentos de defensa de los derechos y el apoyo para la inclusión educativa y social, es hora de llevarlo a efecto. Por ejemplo, en cuanto al incremento de oportunidades y de atención educativa, pero tendrían que ser unas oportunidades reales donde se den las mismas posibilidades de acceso, permanencia, ascenso, graduación, a todos. Y además oportunidades de inclusión que no nieguen la pluralidad en las formas de acceso al mundo y de conocimiento de él.

Por supuesto todas las acciones que se lleven a cabo tendrán que ver con las formas de visibilidad y decibilidad de la diferencia, sólo el lugar desde el cual se les mire, la perspectiva desde la cual se hable de ellos, de sus posibilidades, de sus alcances pondrán los límites, los limitantes o los alcances de estos grupos de población. 


\section{Referencias bibliográficas}

Congreso de Colombia. Constitución Política de 1991. Disponible en: http://www. corteconstitucional.gov.co/inicio/Constitucion\%20politica\%20de\%20Colombia\%20-\%20 2015.pdf

Deleuze, G. (1987). Foucault. Barcelona: Paidós.

Foucault, M. (1999). Gubernamentalidad. En: Estética, ética y hermenéutica. Obras esenciales. Vol. III. Barcelona: Paidós.

Foucault, M. (2000). Los Anormales. México: Fondo de Cultura Económica.

Foucault, M. (2007). Historia de la Sexualidad. Volumen 1 - La Voluntad de Saber. México: Siglo XXI editores.

Unesco. (1996). Informe a la Unesco de la Comisión Internacional sobre la Educación para el Siglo XXI. Presidido por Jacques Delors. Disponible en: http://www.unesco.org/ education/pdf/DELORS_S.PDF

Martínez Boom, A. (2004). De la escuela expansiva a la escuela competitiva. Dos modos de modernización en América Latina. Barcelona: Anthropos.

Ministerio de Educación Nacional, MEN. (1903). Ley 39 de 1903 (26 de octubre) Sobre Instrucción Pública. Publicada por el Diario Oficial 11,931 viernes 30 de octubre de 1903.

Ministerio de Educación Nacional, MEN. (1904). Decreto Número 491 de 1904 (3 de junio) por el cual se reglamenta la Ley 89 de 1903, sobre Instrucción Pública. Publicado por el Diario Oficial 12,122 jueves 14 de julio de 1904.

Ministerio de Educación Nacional, MEN. (1926). Ley 19260012 (septiembre 25) "Sobre enseñanza de la higiene, saneamiento de los puertos marítimos, fluviales y terrestres y de las principales ciudades de la República". Publicada por el Diario oficial 20307 lunes 27 de septiembre de 1926.

Ministerio de Educación Nacional, MEN. (1928). Ley 86 de 1928. "sobre academias nacionales, sociedad geográfica y otras disposiciones sobre instrucción pública". Disponible en: http://www.mineducacion.gov.co/1621/articles-103002_archivo_pdf. pdf

Ministerio de Educación Nacional, MEN. (1930). Decreto 1790 de 1930, por el cual se reglamentan los articulos 4으, 5ㅇ, 6으, 7으, y 8 de la ley 56 de 1927, sobre mínimo de educación obligatoria. Disponible en: http://www.mineducacion.gov.co/1621/ articles-102963_archivo_pdf.pdf 
Ministerio de Educación Nacional, MEN. (1931). Ley 54 de 1931. "por la cual se crean algunos orfanatos, escuelas de artes y oficios, secciones de policía colonizadora y se dan facultades al gobierno para organizar aduanas en las regiones amazónicas". Disponible en: http://www.mineducacion.gov.co/1621/articles-103007_ archivo_pdf.pdf

Ministerio de Educación Nacional, MEN. (1936). Ley 32 DE 1936 (febrero 20) "sobre igualdad de condiciones para el ingreso a los establecimientos de educación" Publicado en Diario Oficial 23127 Bogotá, jueves 5 de marzo de 1936.

Ministerio de Educación Nacional, MEN. (1946). Ley 83 de 1946. Publicada en Diario Oficial No. 26.363, de 24 de febrero de 1947.

Ministerio de Educación Nacional, MEN. (1958). - Oficina de Planeación. Informe del Proyecto para el I Plan Quinquenal. Tomo I. Bogotá. 1958.

Ministerio de Educación Nacional, MEN. (1968). Decreto 3157 de 1968 por el cual se reorganiza el Ministerio de Educación Nacional y se estructura el sector educativo de la Nación. Publicado en Diario Oficial 32697 miércoles 29 de enero de 1969.

Ministerio de Educación Nacional, MEN. (1973). Decreto 2499 de 1973, por el cual se establecen unos cursos de capacitación. Publicado en Diario Oficial 34110 viernes 28 de junio de 1974.

Ministerio de Educación Nacional, MEN. (1974). Decreto 1038 de 1974, por el cual se establecen unos cursos de capacitación. Publicado en Diario Oficial 34110 viernes 28 de junio de 1974.

Ministerio de Educación Nacional, MEN. (1976). Decreto 088 de 1976 por el cual se reestructura el sistema educativo y se reorganiza el Ministerio de Educación Nacional. Publicado en Diario Oficial 34495 lunes de febrero de 1976

Ministerio de Educación Nacional, MEN. (1977). Decreto 0932 de 1977 (mayo 2) por el cual se modifican los Estatutos del Instituto Nacional para Ciegos - INCI

Ministerio de Educación Nacional, MEN. (1978). Decreto 0577 de 1978 (abril 4) por el cual se modifican los estatutos del Instituto Nacional para Ciegos - INCI.

Ministerio de Educación Nacional, MEN. (1978). Decreto 1142 de 1978 (junio 19) por el cual se reglamenta el artículo 118 del Decreto - ley número 088 de 1976 sobre educación de las comunidades indígenas

Ministerio de Educación Nacional, MEN. (1980). Decreto 34 de 1980 (enero 11) por el cual se eximen de cursar idiomas extranjeros a alumnos de Educación Especial. Publicado en Diario Oficial 35449 lunes 4 de febrero de 1980. 
Ministerio de Educación Nacional, MEN. (1980) Decreto 85 de 1980 (enero 23) por el cual se introducen unas modificaciones al Decreto extraordinario 2277 de 1979. Publicado en: Diario Oficial miércoles 27 de febrero de 1980.

Ministerio de Educación Nacional, MEN. (1981). Decreto 3486 de 1981 (diciembre 9) por el cual se establece el régimen de matrículas y pensiones, becas, Juntas Reguladoras de Matrículas y Pensiones y otras variables de costos en los establecimientos educativos no oficiales de los niveles preescolar, básica (primaria y secundaria), media vocacional y educación especial, y se dictan otras disposiciones.

Ministerio de Educación Nacional, MEN. (1986). Decreto 2230 de 1986 (julio 14) por el cual se crea el Comité Nacional de Lingüística Aborigen.

Ministerio de Educación Nacional, MEN. (1986). Decreto 0428 de 1986 (febrero 7) Por el cual se establece el Plan de Estudios para la Educación Básica Primaria de Adultos

Ministerio de Educación Nacional, MEN. (1986). Decreto 1895 de 1986 (junio 13) por el cual se reglamenta el artículo 20 de la Ley 20 de 1982, que regula el Fondo Especial de Capacitación de los trabajadores menores de dieciocho (18) años de edad, por parte del Servicio Nacional de Aprendizaje, SENA.

Ministerio de Educación Nacional, MEN. (1994). Ley 115. General de Educación de febrero 8 de 1994. Disponible en: http://www.mineducacion.gov.co/1621/ articles-85906_archivo_pdf.pdf

Ministerio de Educación Nacional, MEN. (2005). Lineamientos de política para la atención educativa a poblaciones vulnerables. Disponible en: http://www. mineducacion.gov.co/cvn/1665/article-90668.html

Pardo, J.L. (1990). Deleuze: Violentar el pensamiento. Madrid: Editorial Cincel.

Unesco. (1994). Conferencia Mundial sobre Necesidades Educativas Especiales: Acceso y Calidad - Salamanca: Unesco. Disponible en: http://unesdoc.unesco.org/ images/0011/001107/110753so.pdf

Zourabichvili, F. (2004). Deleuze. Una filosofía del acontecimiento. Buenos Aires: Amorrortu. 